\title{
Developing the loT through wireless communication networks: analysis of topologies
}

\begin{abstract}
Standard structure of Wi-Fi networks is usually a communication manager and a number of clients. In this basic system, clients will always listen for the presence of one or more managers who tell them, among other information, the name of the network that they manage, the channel to use, the security and available authentication algorithms, etc. Nowadays, wireless networks can be classified into two general categories: infrastructure-based wireless networks and infrastructure-less wireless networks, or more commonly, ad hoc wireless networks. Different topologies can be used with any purpose of communication among a network of nodes and in this paper several of them are analyzed.
\end{abstract}

Keywords: zigbee, wireless network, network topology, protocol, communication
Volume 3 Issue 4 - 2017

\author{
Javier Bilbao, Eugenio Bravo, Concepción \\ Varela, Olatz García, Carolina Rebollar \\ Applied Mathematics Department, University of the Basque \\ Country, Spain
}

Correspondence: Javier Bilbao, Applied Mathematics Department, University of the Basque Country, UPV/EHU, Engineering School, Alda Urkijo, s/n, 480I3-Bilbao, Spain, Email javier.bilbao@ehu.es

Received:September 30, 2017| Published: November 15, 2017

\section{Introduction}

Sensor networks consist of a set of sensor nodes which are spread over a geographical area. These nodes are able to perform processing as well as sensing and are additionally capable of communicating with each other. ${ }^{1}$ Topology control is one primary challenge in these scenarios. Unlike the wired networks that typically have fixed network topologies, each sensor node in a sensor network can potentially change the network topology by adjusting its transmission range and/ or selecting specific nodes to forward its messages, thus, controlling its set of neighbors. ${ }^{1}$ This is very important because future Internet aims to integrate heterogeneous communication technologies, both wired and wireless, in order to contribute substantially to assert the concept of Internet of Things (IoT). ${ }^{2}$ At the same time, prices of sensors, microprocessors, open-hardware and other electronic devices that support the IoT are low, and, in consequence, there is a proliferation of Wireless Sensor Networks (WSN) in many applicative scenarios.

Some examples include monitoring and control of industrial processes, monitoring medical applications, monitoring of environmental variables, home automation, traffic control, etc. ${ }^{3,4}$ WSN or systems based on them are proposed during the last years as projects and some of them become reality, such as Rampe system that describes a system for the mobility and autonomy of blind people in public transports. ${ }^{5}$ It could be also employed for elder, disabled or wheelchair passengers. ${ }^{6}$ Lavanya et al. ${ }^{7}$ presented in 2013 another project where Wireless Sensor Networks were used with the aim of helping blind people for independent navigation in urban transport is presented in. ${ }^{7}$ In this case, the system was based on ZigBee technology. WSN are made up by autonomous distributed nodes. The nodes include sensors to monitor physical or environmental parameters and a radio transceiver for transmitting and receiving information. These networks are based on packet switching, so that each block of information is fragmented into transmission small units called packets. ${ }^{8-10}$ The nodes must be very simple in hardware and user interface components. In addition to be equipped with one or more sensors, each node features:

i. A radio transceiver or other wireless communication module.

ii. A low-power microcontroller. iii. A battery.

The nodes of this kind of networks must be robust to allow its implementation in adverse environments, they must have low power consumption and manufacturing must be economical. Regarding to the communications module (radio transceiver), IEEE 802.15.4 2006 standard defines the medium-access control layer (MAC) and the physical layer (PHY). This is the standard used by ZigBee. It is a specification for low-power mesh networks, promoted by a broad consortium of industry promoters (ZigBee Alliance). ZigBee includes IEEE 802.15.4 standard for the lower layers of the protocol stack. ${ }^{11}$

\section{Basics on topology and MANETs}

Topology control has drawn significant research interest, ${ }^{12-19}$ in last few years. Different topologies have different properties; however, none of them can achieve all three preferred properties for unicast applications on wireless ad hoc networks: power spanner, planar, degree-bounded. Mobile ad-hoc networks, known as MANETs, represent a great potential for communication, interaction, and collaboration in a world with a rapidly increasing number of electronic devices everywhere, from computers to smartphones or any device with a microprocessor and wireless connection. Until first years of this century, Wang et al..$^{20}$ proposed a localized algorithm to build a degree-bounded planar spanner both in centralized and distributed way, which is based on the combination of localized Delaunay triangulations $(\mathrm{LDel})^{21}$ and $\mathrm{YaO}^{22}$ structure. It is the first localized algorithm that can achieve all the three desirable features. However, the node degree of their structure can reach 25 in the worst case; and the communication cost of their method can be large, although it is shown that the total number of messages is $\mathrm{O}(\mathrm{n})$, the hidden constant could be as high as several hundred since the method needs to collect the 2-hop information for every node. ${ }^{23}$

\section{IEEE 802.| 5.4 standard}

IEEE 802.15.4 standard defines the specifications of the physical layer (PHY) and of the medium-access control (MAC) layer for wireless connections with low data rate, in single low-power nodes operating in a usually range coverage of 10 meters. Therefore, it is a standard designed specifically for low power wireless personal area 
networks (LR-WPAN). ZigBee is one of the newest technologies enabling Wireless Personal Area Networks (WPAN). ZigBee is the name of a specification for a suite of high level communication protocols using small, low-power digital radios based on the IEEE 802.15.4 standard. The technology is intended to be simpler and cheaper than other WPANs, such as Bluetooth. ZigBee protocols are intended for use in embedded applications requiring low data rates and low power consumption. Though WPAN implies a reach of only a few meters, normally 10 meters, the network will have several layers, so designed as to enable intrapersonal communication within the network, connection to a network of higher level and ultimately an uplink to internet. The typical ZigBee transmission range is roughly 50 meters. The ZigBee Alliance is providing a standardized base set of solutions for sensor and control systems. To allow vendors to supply the lowest possible cost devices the IEEE standard defines two types of devices: full function devices (FFD) and reduced function devices (RFD). ${ }^{24}$ An IEEE 802.15.4/ZigBee network requires at least one full function device as a network coordinator, but endpoint devices may be reduced functionality devices to reduce system cost. The ZigBee Standard has evolved standardized sets of solutions, called 'layers'. ${ }^{24}$ These layers facilitate the features that make ZigBee very attractive: low cost, easy implementation, reliable data transfer, short-range operations, very low power consumption and adequate security features. ZigBee is very similar to Bluetooth but with some differences and advantages for home automation:

i. A ZigBee network can consist of a maximum of 65535 nodes distributed in subnetworks of 255 nodes, compared to eight maximum of one subnet (Piconet) of Bluetooth.

ii. It has lower power consumption than Bluetooth. In exact terms, ZigBee has a consumption of $30 \mathrm{~mA}$ in transmission mode and $3 \mu \mathrm{A}$ at rest, against $40 \mathrm{~mA}$ in transmission mode and $0.2 \mathrm{~mA}$ at rest that Bluetooth has. This lower consumption is because the ZigBee system most of the time stays asleep while on a Bluetooth communication this is not possible and it is always transmitting and / or receiving.

iii. It has a speed of $250 \mathrm{kbps}$, whereas in Bluetooth is up to 3000 kbps, normally 1 Mbps.

iv. Because of the speed of these protocols, each one is more appropriate than the other for certain things.

For example, while Bluetooth is used for applications such as mobile phones and home computers, the speed of the ZigBee becomes insufficient for these tasks, diverting it to applications such as home automation, dependent battery products, medical sensors, articles and toys, in which data transfer is lower. Three main characteristics of ZigBee that have to be taken into account are the following:

Network and application support layer: The network layer has been designed to allow the network to spatially grow without requiring high power transmitters. The network layer also can handle large amounts of nodes with relatively low latencies. The APS sub-layer's responsibilities include maintenance of tables that enable matching between two devices and communication among them, and also discovery, the aspect that identifies other devices that operate in the operating space of any device.

Physical layer: The IEEE 802.15.4 physical layer accommodates high levels of integration by using direct sequence to permit simplicity in the analog circuitry and enable cheaper implementations.

Media access control layer: The IEEE 802.15.4 media access

control layer permits use of several topologies without introducing complexity and is meant to work with large numbers of devices.

\section{Network topologies}

One of the great advantages of the IEEE 802.15.4 standard is the ability to create multiple alternative paths that will give robustness to any adversity through a mesh network. Although this type of topology is the most commonly used, there are others that are discussed below and can be seen in Figure 1.

a)

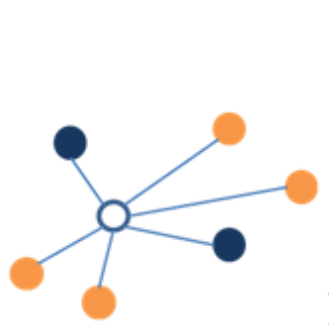

b)

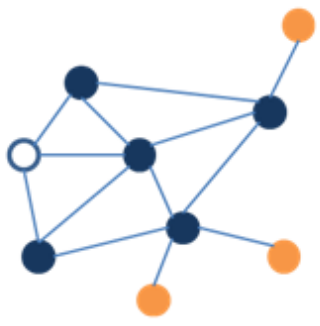

c)

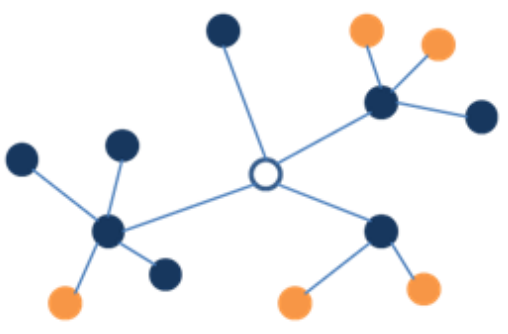

Figure I Network topologies:A) star, B) mesh, C) cluster-tree.

\section{Star}

Communications always go through the hub (central node), which acts as PAN (Personal Area Network) coordinator. Such networks are quite vulnerable because they depend on the proper functioning of the central node. Before the fall of the coordinator, it is essential to have in place a system for the network to be reconfigured again.

\section{Mesh}

As mentioned before, it is the preferred topology for the ZigBee technology. Multiple paths are created within the network that give robustness to the fall of one or more nodes. The grid structure can be complemented with small branches, hanging nodes with other or reduced functionality.

\section{Cluster-tree}

This topology encompassing the previous two topologies. A series of clusters (group of RFD -Reduced Function Device- nodes) are created where a FFD node (Full Function Device) adopts the role of coordinator of the cluster. Each cluster coordinator is responsible for communicating with all those nodes that has directly connected, creating a star arrangement. As a complement, if the vertices of the star are FFD, these can be linked with RFD, as it happens in the mesh topology.

\section{CSMA/CA}

The main function of the MAC layer is to manage access to the medium. The aim is that all nodes of the network share in an efficient and fair manner the transmission medium, in this case the radio channel [25]. The basis of the MAC protocol defined in the 
IEEE 802.15.4 is the CSMA/CA (Carrier Sense Multiple Access with Collision Avoidance) algorithm, that is, listen before talk (LBT). In the standard, two types of CSMA/CA are defined whose application depends on whether you opt for access control based on beacons or not:

Mode without beacon: Based on unslotted CSMA/CA access.

Beacon-enabled mode: Based on slotted CSMA/CA access.

\section{Nonbeacon-enabled unslotted CSMA/CA network}

In a network without beacons unslotted CSMA/CA is used. Each time a node wants to perform transmission of a packet, it must wait a random time, called backoff time, and during this time it does not hear the channel status. When this time has passed, the channel is heard. If the channel is free (there is no transmission in this moment), the node can transmit data. If the channel is busy, the node must wait another random period before trying to access the channel again. Figure 2 shows the flowchart of the medium access protocol. In this process, some variables are involved:

i. NB: Counter of the number of times the backoff is executed to access the medium in each transmission attempt of a packet.

ii. BE: Is the backoff exponent, which is related to the backoff time that the node waits before evaluating the state of the channel. Here it is assigned as the initial value macMinBE, where the value recommended in the IEEE 802.15.4 standard is 3 .

iii. $\mathbf{a M a x B E}=$ Maximum permissible value for the variable $\mathrm{BE}$. According to the standard default is 5 . With this variable the maximum backoff time that the node waits before evaluating the channel is specified.

iv. macMaxCSMABackoffs=Maximum permissible value for the value of the NB variable, that is, the maximum number of transmission attempts. The standard defines the default value of 4 .

\section{Beacon-enabled slotted CSMA/CA network}

For networks with beacon mode activated, the channel access slotted CSMA/CA is used. In this case, the backoff periods are synchronized with the start of the transmission of a beacon. The use of beacons adds a new level of functionality to the MAC layer of the network. Nodes can be in off mode and wake up only when they receive a beacon signal, listen to its direction and return to the sleep mode, thereby saving energy. The beacon frames are important to keep all nodes synchronized without requiring nodes consume energy listening for long periods of time. In the Figure 3 the structure of a beacon frame is shown. The most important fields are in the MAC payload, which contains: super frame specification, GTS fields, pending address fields, and the beacon payload. The beacon-enabled mode is often used in networks of star topology, where a coordinator can create a super frame structure, defined by the regular transmission of beacons.

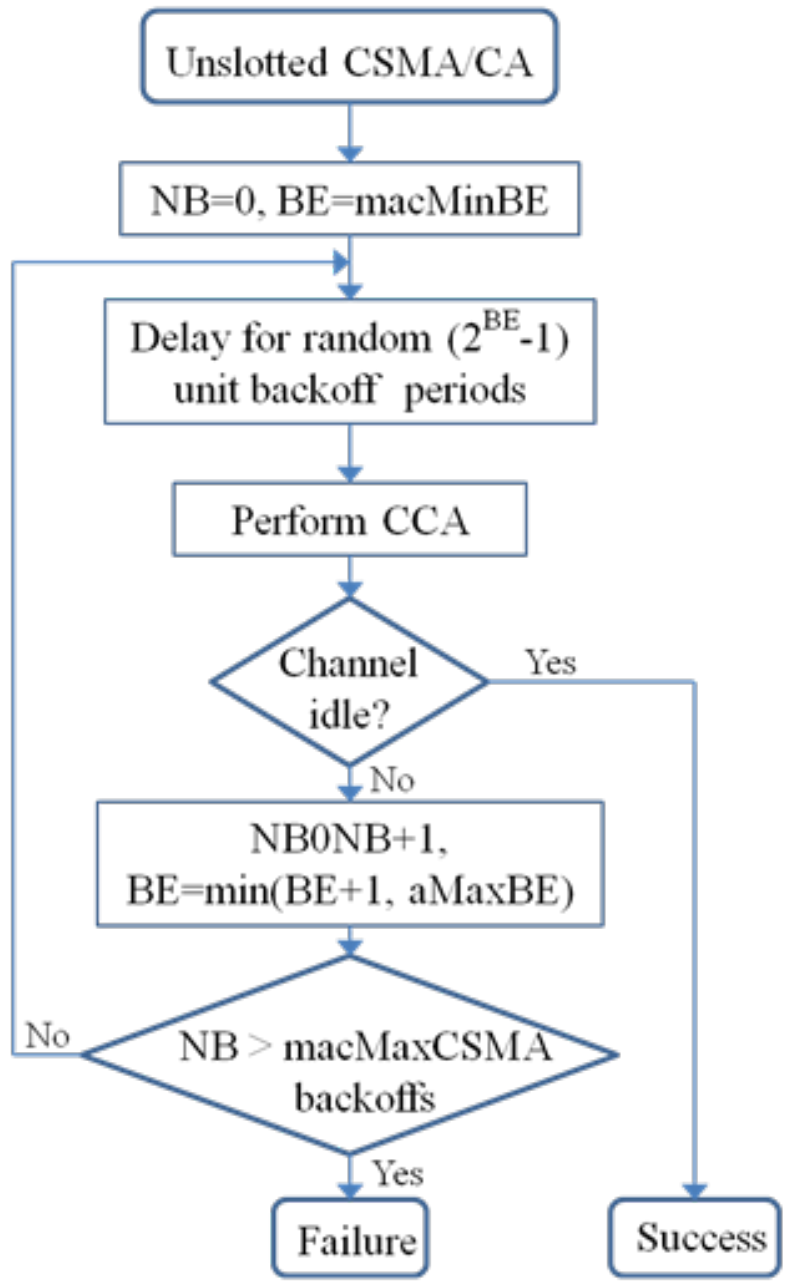

Figure 2 Unslotted CSMA/CA algorithm.

\begin{tabular}{|c|c|c|c|c|c|c|c|c|c|c|c|c|}
\hline Oetets: 2 & 1 & \multicolumn{2}{|l|}{4 or 10} & \multicolumn{2}{|c|}{2} & \multicolumn{2}{|c|}{ variable } & \multicolumn{2}{|c|}{ variable } & \multicolumn{2}{|c|}{ variable } & 2 \\
\hline $\begin{array}{l}\text { Frame } \\
\text { control }\end{array}$ & $\begin{array}{c}\text { Bescon } \\
\text { sequence } \\
\text { number }\end{array}$ & \multicolumn{2}{|c|}{$\begin{array}{l}\text { Source address } \\
\text { information }\end{array}$} & \multicolumn{2}{|c|}{$\begin{array}{l}\text { Superframe } \\
\text { specification }\end{array}$} & \multicolumn{2}{|c|}{$\begin{array}{l}\text { GTS } \\
\text { fields }\end{array}$} & \multicolumn{2}{|c|}{$\begin{array}{l}\text { Pending } \\
\text { address } \\
\text { ficlds }\end{array}$} & \multicolumn{2}{|c|}{ Beacon paylosd } & $\begin{array}{c}\text { Frame } \\
\text { check } \\
\text { sequence }\end{array}$ \\
\hline \multicolumn{4}{|c|}{ MAC header } & \multicolumn{8}{|c|}{ MAC payload } & $\begin{array}{l}\text { MAC } \\
\text { footer }\end{array}$ \\
\hline & Bits: 0.3 & $4-7$ & & 11 & 12 & & & 3 & & 14 & 15 & \\
\hline & $\begin{array}{l}\text { Beacon } \\
\text { order }\end{array}$ & $\begin{array}{l}\text { Superframe } \\
\text { order }\end{array}$ & $\begin{array}{r}\text { Fina } \\
s\end{array}$ & $\begin{array}{l}\text { ICAP } \\
\text { lot }\end{array}$ & $\begin{array}{l}\text { Batter } \\
\text { extens }\end{array}$ & $\begin{array}{l}\text { ylife } \\
\text { sion }\end{array}$ & Rest & rved & $\begin{array}{r}f \\
\text { coos }\end{array}$ & $\begin{array}{l}\text { AN } \\
\text { dinator }\end{array}$ & $\begin{array}{c}\text { Association } \\
\text { permit }\end{array}$ & \\
\hline
\end{tabular}

Figure 3 Structure of the beacon frame. 


\section{Conclusion}

When standardization was created, following the creation of the "Wi-Fi Alliance" in 1996, an organization that was born with the intention to verify, certify and promote systems to 802.11 , Wireless Communication Networks began to be used by the general public. In Wi-Fi networks, it always exists, as a basic structure, a communication manager and a number of customers. Customers always listen for the presence of one or more managers who tell them, among other information, the name of the network to manage the channel to use, security and authentication algorithms available, etc. Based on this information and settings of the device of the network, the customer will be able to join the appropriate network. Depending on who implements the function of network management, we will face an "adhoc" network, in which the manager is a member of the same network computer/device, or a network of type "infrastructure", where the manager is an access point, router or similar. Most wireless nodes that operate with the IEEE 802.15.4 standard implements the unslotted CSMA/CA method as medium access (MAC) protocol. This MAC protocol offers high performance in environments with low traffic load and few nodes, providing good response in terms of transmission rate (throughput) and in terms of medium access delay (delay). However, its performance is seriously harmed when the number of nodes competing for the channel is high, or when the traffic load is high. In addition, number of wireless nodes in the market is growing up continuously and the appearance of new multimedia services and applications has resulted in wireless networks become saturated with nodes that require higher transmission speeds. On the other hand, CSMA-based protocols are not commonly energy efficient, and Machine to Machine (M2M) applications, with a boom at present, require the operational and autonomous life of WSN is as long as possible in order to avoid the battery replacement cost. Therefore, it should be ideal to design new MAC protocols that provide good performance for any number of nodes and for high traffic loads, always offering energy efficiencies that ensure long lifetime in such networks. There are currently numerous proposals for MAC protocols for ad hoc networks that improve the performance of CSMA/CA. DPCF MAC (Distributed Point Coordination Function) protocol is one of these proposals. It is a MAC protocol designed to work on wireless ad hoc environments. The main design goal of this protocol is to increase the performance of these networks in terms of throughput and transmission delay.

\section{Acknowledgements}

None.

\section{Conflict of interest}

The author declares no conflict of interest.

\section{References}

1. Yu Wang. Topology control for wireless sensor networks, topology control for wireless sensor networks. In: Li Y, et al. editors. Wireless Sensor Networks and Applications. USA: Springer; 2008. p. 113-147.

2. Kortuem G, Kawsar F, Sundramoorthy V, et al. Smart objects as building blocks for the Internet of things. IEEE Internet Computing. 2009;14(1):44-51.

3. Mercilin Raajini X, Raja Kumar R, Indumathi P. Fuzzy based faulty link isolation technique in dynamic wireless sensor networks. WSEAS Transactions on Computers. 2015;14:97-106.
4. Curiac DI, Volosencu C, Pescaru D, et al. Redundancy and its applications in wireless sensor networks: a survey. WSEAS Transactions on Computers. 2009;8(4):705-714.

5. Baudoin G, Venard O, Uzan G, et al, How can blinds get information in public transports using PDA? The RAMPE Auditive man machine interface, $8^{\text {th }}$ Proceeding AAATE; France: Springer; 2005. p. 304-316.

6. Leire Azpilicueta, Peio López Iturri, Erik Aguirre, et al. Characterization of Wireless Channel Impact on Wireless Sensor Network Performance in Public Transportation Buses. IEEE Transactions on Intelligent Transportation Systems. 2015;16(6):3280-3293.

7. Lavanya G, Preethy W, Shameem A, et al. Passenger BUS alert system for easy navigation of blind. International conference on circuits; Power and computing technologies. India: IEEE; 2013.

8. Akkaya K, Younis M. A survey on routing protocols for wireless sensor networks. Ad hoc networks. 2005;3(3):325-349.

9. Liu G, Xu B, Chen H. Decentralized estimation over noisy channels in cluster-based wireless sensor networks. International Journal of Communication Systems. 2012;25(10):1313-1329.

10. Mardini W, Yassein MB, Khamayseh Y, et al. Rotated hybrid, energyefficient and distributed (R-HEED) Clustering protocol in WSN. WSEAS Transactions on Communications. 2014;3:275-290.

11. Part 15.4: Wireless Medium access control (MAC) and physical layer (PHY) Specifications for low-rate wireless personal Area networks (WPANs). USA: IEEE; 2006.

12. Grünewald M, Lukovszki T, Schindelhauer C, et al. Distributed maintenance of resource efficient wireless network topologies. European Conference on Parallel Processing; Germany: Springer; 2002. p. 935946.

13. Li Li, Joseph Y Halpern, Paramvir Bahl, et al. Analysis of a cone-based distributed topology control algorithms for wireless multi-hop networks. IEEE/ACM Transactions on Networking. 2001;13(1):147-159.

14. Xiang-Yang Li, Peng-Jun Wan, Yu Wang. Power efficient and sparse spanner for wireless ad hoc networks. Computer communications and networks, $10^{\text {th }}$ International Conference; USA: IEEE; 2001.

15. Xiang-Yang Li, Peng-Jun Wan, Yu Wang, et al. Sparse power efficient topology for wireless networks. System Sciences, Proceedings of the $35^{\text {th }}$ Annual hawaii international conference; USA: IEEE; 2002.

16. Rajmohan Rajaraman. Topology control and routing in ad hoc networks: A survey. SIGACT News. 2002;33(2):60-73.

17. Ramanathan R, Rosales-Hain R. Topology control of multihop wireless networks using transmit power adjustment. Nineteenth Annual Joint Conference of the IEEE computer and communications societies; Israel: IEEE; 2000.

18. Yu Wang, Xiang-Yang Li, Ophir Frieder. Distributed spanner with bounded degree for wireless networks. International Journal of Foundations of Computer Science. 2003;14(1):183.

19. Roger Wattenhofer, Li Li, Paramvir Bahl, Yi-Min Wang. Distributed topology control for wireless multihop ad-hoc networks; 2000.

20. Yu Wang, Xiang-Yang Li. Localized construction of bounded degree planar spanner for wireless networks. DIALM-POMC '03 Proceedings, USA: IEEE; 2003. p. 59-68.

21. Xiang-Yang Li, Calinescu G, Peng-Jun Wan. Distributed construction of planar spanner and routing for ad hoc wireless networks. $21^{\text {st }}$ Annual Joint Conference; USA: IEEE; 2002.

22. Yao ACC. On constructing minimum spanning trees in k-dimensional spaces and related problems. SIAM J Computing. 1982;11:721-736. 
23. Wen-Zhan Song, Yu Wang, Xiang-Yang Li, et al. Localized algorithms for energy efficient topology in wireless ad hoc networks. Japan: ACM; 2004.

24. Kinney P. ZigBee Technology: wireless control that simply works. Communications design conference; 2003.
25. Vahabi M, Fotouhi Ghazvini M. Energy Efficiency in MAC 802.15.4 for wireless sensor networks. Malaysia: IEEE; 2008. 